\title{
COMMUNICABLE DISEASES REPORT, NSW, FOR JULY AND AUGUST 2004
}

For information on communicable diseases in New South Wales that is updated regularly, visit www.health.nsw.gov.au and click on Infectious Diseases.

\section{TRENDS}

Tables 1 and 2 show reports of notifiable conditions for each area health service in NSW received through to the end of July and August 2004. Figure 1 presents trends in reports of communicable diseases for the period 1999 through to the end of August. Note that due to an upgrade in the Notifiable Disease Database, some data entry for cases reported in July may have been delayed resulting in some underreporting of cases for that month.

Analysis of recent case notifications shows a typical late winter pattern of communicable diseases in NSW: low rates of arboviral infections, cryptosporidiosis, and salmonellosis; relatively higher rates of meningococcal disease (see www.health.nsw.gov.au/public-health/alerts/ meningococcal/men_update.html) and pneumococcal disease; and increasing pertussis. One case of cholera was reported in August in a person who had travelled to the Philippines.

In what appears to be a late influenza season, relatively little influenza activity was reported through to August. Reports from selected general practitioners and laboratories indicate a modest rise towards the end of the month (see www.health.nsw.gov.au/living/flureport.html for a detailed report). Influenza infections are likely to increase further in September.

The epidemic of diarrhoea and vomiting linked to norovirus that was evident in late autumn and early winter seems to have abated. Reports of outbreaks across NSW in institutions (mainly nursing homes and some hospitals) peaked in May with 100 outbreaks reported that involved a total of over 3,294 ill people. By the end of August, 17 outbreaks had been reported for that month involving a total of 184 ill people.

\section{HEPATITIS A IN A SYDNEY FOOD HANDLER}

The Central Sydney and South Eastern Sydney Public Health Units reported that a food handler working in a Sydney café was diagnosed with hepatitis A in July. Hepatitis A is an acute viral infection of the liver. It is characterised by fever, feeling unwell, poor appetite, abdominal discomfort, and jaundice. The virus is transmitted by the faecal-oral route, through direct contact with an infectious person or the ingestion of contaminated food or contaminated water.

Normal human immunoglobulin is a purified blood product that contains antibodies to the hepatitis A virus. An injection of normal human immunoglobulin can prevent illness if given soon after and within two weeks of exposure to the virus.

While infectious with hepatitis A, the food handler was involved in slicing fresh salad vegetables for sandwiches. The food handler was unaware of their infection at the time. The food handler and other workers at the café, were interviewed and the café was assessed by food inspectors. A risk assessment indicated that there was a small but significant risk that patrons who ate foods that contained raw salad ingredients could contract hepatitis A. NSW Health issued a media release to alert patrons to this risk, a recorded message hotline was set up, and the café proprietors were asked to distribute flyers to all patrons who attended the café. The South Eastern Sydney Public Health Unit provided a clinic located at Sydney Hospital to counsel patrons at risk and administer normal human immunoglobulin for eligible patrons. The hotline received about 280 calls. At Sydney Hospital, 138 patrons were counselled and 91 patrons received normal human immunoglobulin.

Many of the café patrons who attended the clinic reported multiple possible exposures over the 19-day period in which the food handler was working while infectious. As of 24 August, no secondary cases of hepatitis A have been identified related to the consumption of food from the café. In an unrelated outbreak, four secondary cases were reported among people who ate food prepared by a food handler with hepatitis A in Sydney in late 2003 (see 'A food handler diagnosed with hepatitis A' at www.health.nsw.gov.au/public-health/phb/HTML2004/ march04html/cdrp44.htm).

\section{SALMONELLOSIS OUTBREAK IN A MID NORTH COAST RESIDENTIAL FACILITY}

The Mid North Coast Public Health Unit reported an outbreak of Salmonella Typhimurium 135 infection at a residential facility in June 2004. Of the 57 people who worked or lived at the facility, 43 (75 per cent) became ill with vomiting, diarrhoea, and fever. Symptoms lasted between eight hours and 11 days. Twenty-one people, including two children, were admitted to hospital, four of whom developed septicaemia. The hospital staff reported the outbreak to the Mid North Coast Public Health Unit.

Eighteen stool samples were obtained. Salmonella was identified in all samples; rotavirus was not detected. Stool samples were not tested for norovirus.

Reliable food histories could not be obtained from the residents. However, investigation by the Mid North Coast Public Health Unit suggested that transmission of Salmonella was initially due to person-to-person contact, based on the onset of the initial cases ranging over several 
days. A subsequent rapid rise in cases suggested that consumption of contaminated food was responsible for transmission between people later in the outbreak. Further investigation found that a neighbour of one of the facility's food-handlers became ill after consuming custard prepared at the facility. A stool sample from the neighbour and a sample of the remaining custard from the facility were also found to contain Salmonella Typhimurium 135.

The NSW Food Authority and the Mid North Coast Public Health Unit jointly assessed the facility's hygiene and food handling procedures. They identified a lack of hand washing facilities and evidence of rodent infestation. The facility had no food safety plan and no training for food handlers was provided.

To halt the spread of the bacteria, the Mid North Coast Public Health Unit advised that: residents and staff practice careful hand-washing; environmental cleaning be enhanced; group activities among the residents cease; ill residents be isolated from others; meals be taken in individual rooms; and the facility be closed to admissions for the duration of the outbreak. Food handlers with symptoms of salmonellosis were excluded from work until Salmonella was no longer detected in their stool samples (as per Salmonellosis Draft Response Protocol for NSW public health units at www.health.nsw.gov.au/living/ salmonella.pdf).

Although in this outbreak the pre-existing poor health status of many residents may have contributed to the high attack rate and the severity of illness, outbreaks of salmonellosis in any setting can be severe. Early identification and intervention are vital for the successful control of outbreaks. Such outbreaks may be avoided or contained through improved knowledge among staff of safe food handling procedures, and early reporting of outbreaks by facility managers and attending medical practitioners. 


\section{FIGURE 1}

\section{REPORTS OF SELECTED COMMUNICABLE DISEASES, NSW, JAN 1999 TO AUG 2004, BY MONTH OF ONSET}

Preliminary data: case counts in recent months may increase because of reporting delays.

Laboratory-confirmed cases only, except for measles, meningococcal disease and pertussis $\mathrm{BFV}=$ Barmah Forest virus infections, RRV = Ross River virus infections

lab $+=$ laboratory confirmed
Men Gp C and Gp B = meningococcal disease due to serogroup $C$ and serogroup $B$ infection, other/unk = other or unknown serogroups. NB: multiple series in graphs are stacked, except gastroenteritis outbreaks.

NB. Outbreaks are more likely to be reported by nursing homes \& hospitals than from other institutions

\begin{tabular}{|rc|}
\hline \multicolumn{3}{|c|}{ NSW population } \\
Male & $50 \%$ \\
$<5$ & $7 \%$ \\
$5-24$ & $28 \%$ \\
$25-64$ & $52 \%$ \\
$65+$ & $13 \%$ \\
Rural $^{*}$ & $42 \%$ \\
\hline
\end{tabular}

\begin{tabular}{|rc|}
\hline \multicolumn{2}{|c|}{ July 04-Aug 04 } \\
Male & $51 \%$ \\
$<5$ & $1 \%$ \\
$5-24$ & $9 \%$ \\
$25-64$ & $77 \%$ \\
$65+$ & $13 \%$ \\
Rural & $96 \%$ \\
\hline
\end{tabular}

cases

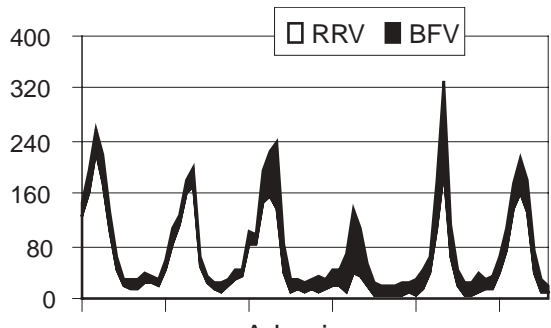

Arbovirus
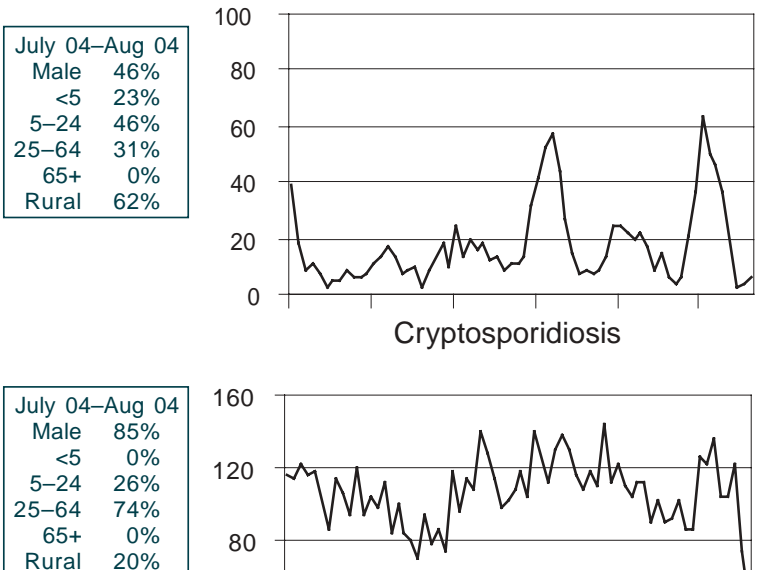

\begin{tabular}{rr} 
Rural & $0 \%$ \\
\hline
\end{tabular}
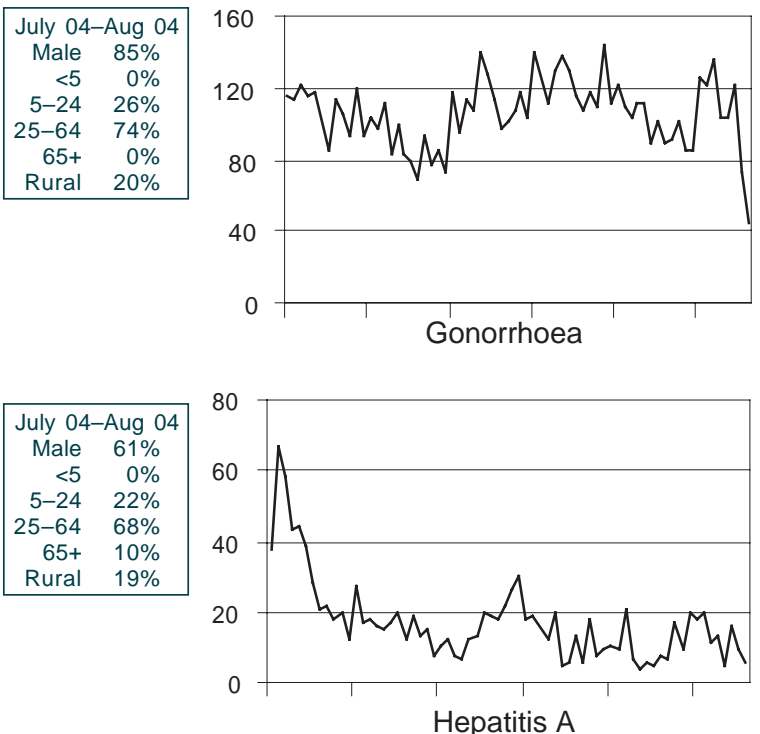

\begin{tabular}{|lr|}
\hline July $04-$ Aug & 04 \\
All outbreaks & 188 \\
Nursing homes & 139 \\
Hospitals & 33 \\
Child care & 12 \\
Schools & 1 \\
Other & 3 \\
\hline
\end{tabular}

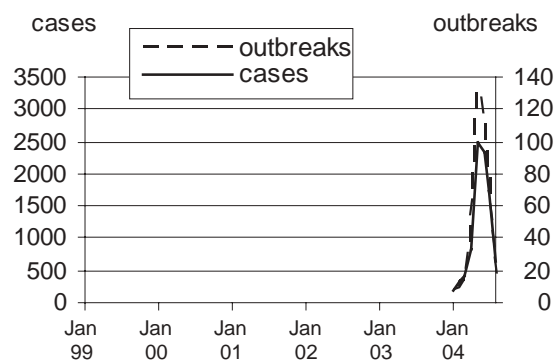

Gastroenteritis outbreaks in institutions
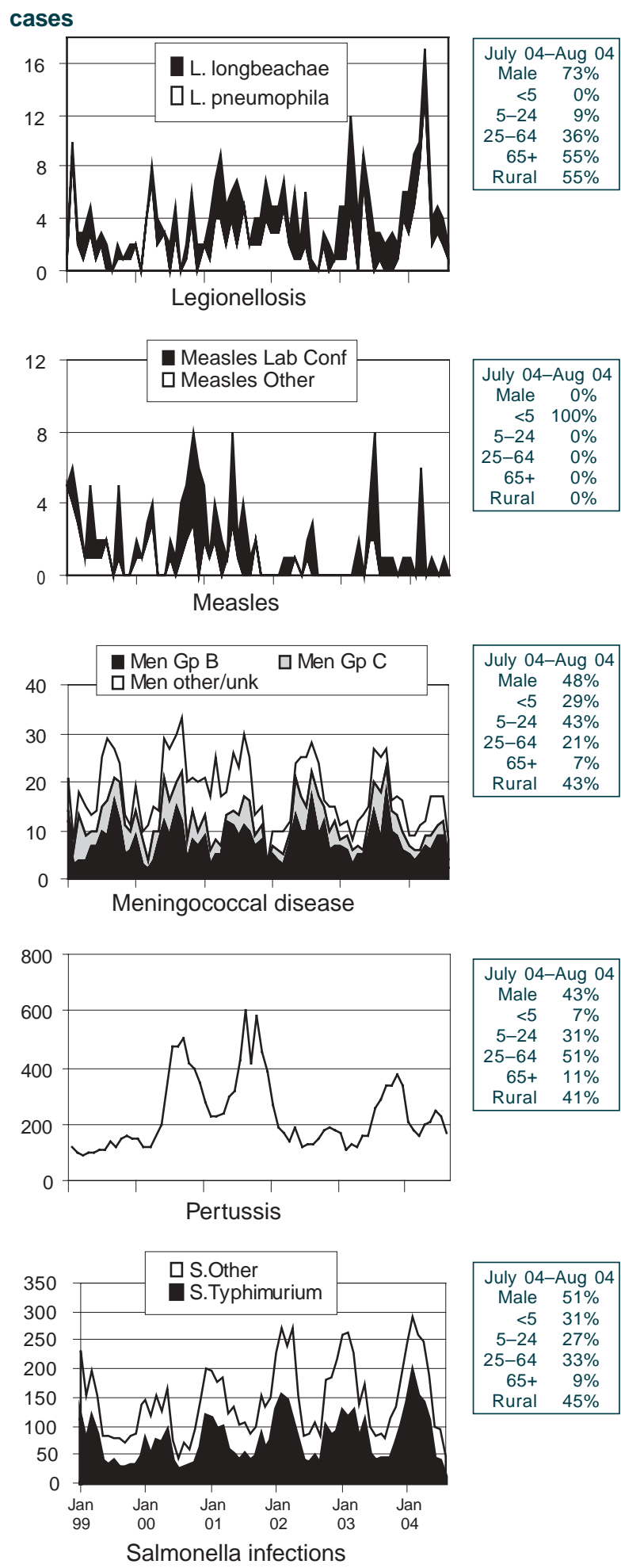


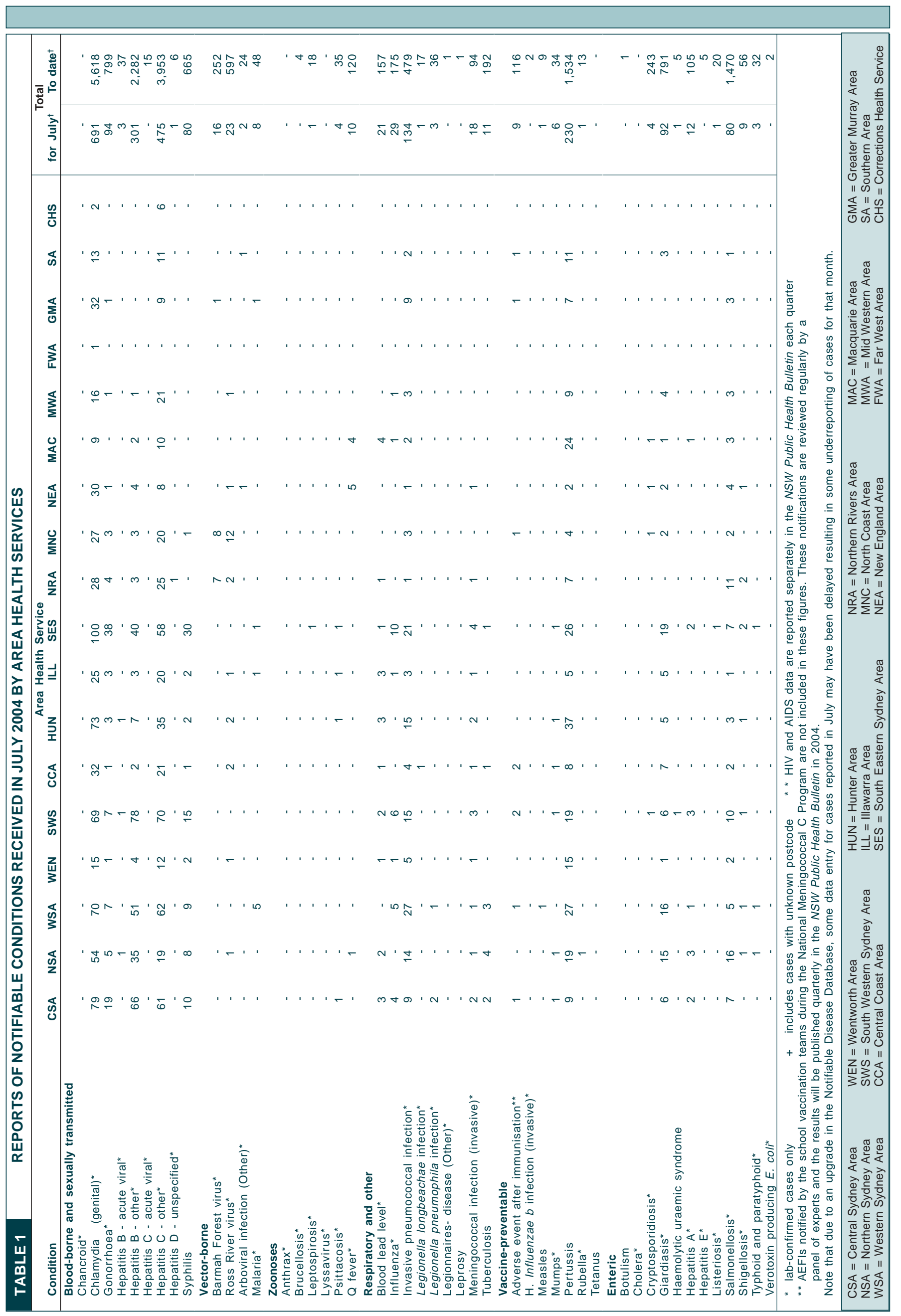




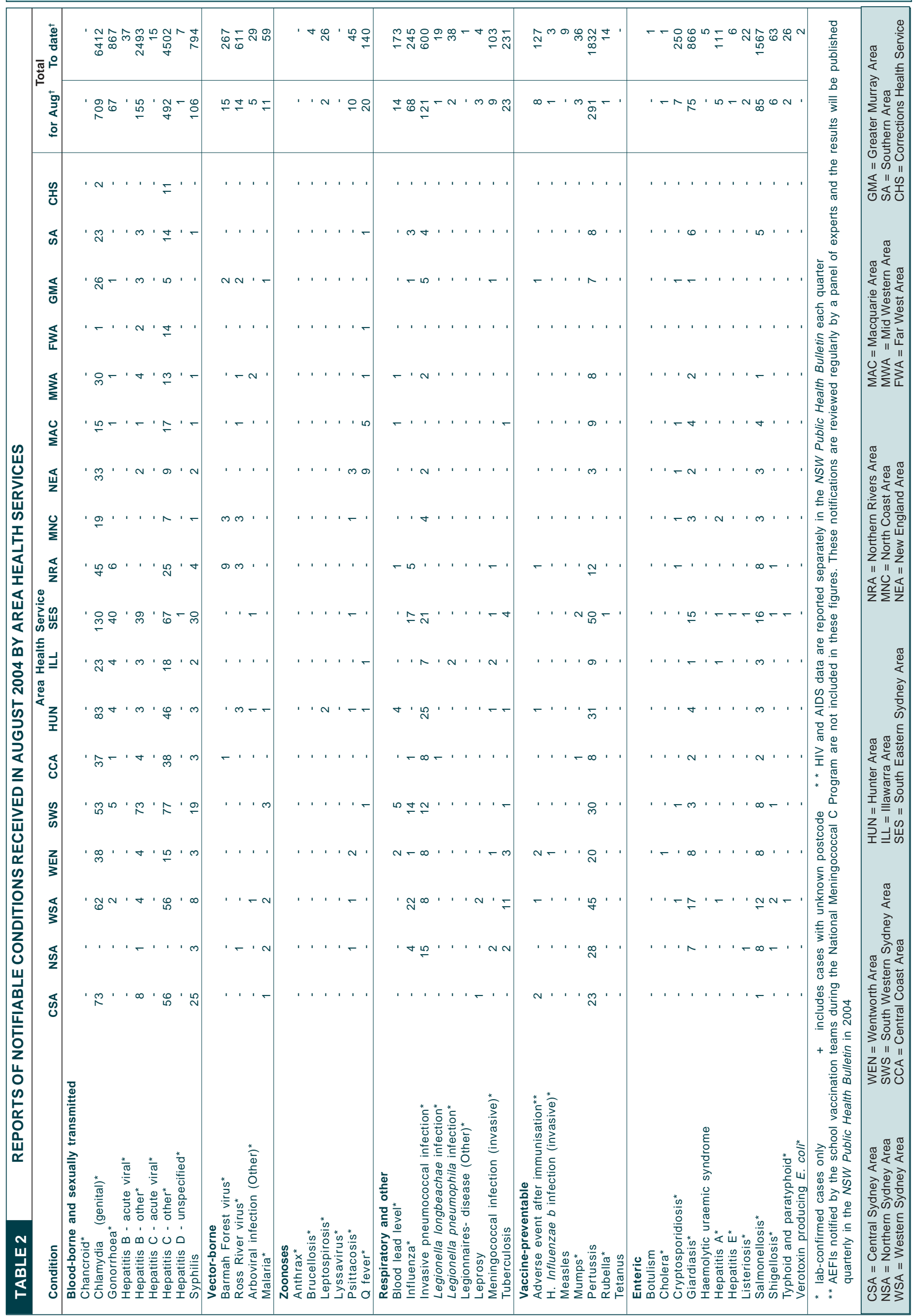

